\title{
CARACTERIZAÇÃO DE TALCO PURIFICADO DE RESÍDUOS DE PEDRA SABÃO
}

\author{
N. H. GARCIA ${ }^{1}$, M. M. L. RODRIGUES ${ }^{2}$, M. M. F. LIMA ${ }^{3}$ e R. M. F. LIMA ${ }^{4}$ \\ Universidade Federal de Ouro Preto \\ neylor_henrique@hotmail.com ${ }^{1}$ - mitchel.marques@yahoo.com ${ }^{2}$ - margarida@em.ufop.br ${ }^{3}$ - \\ rosa@demin.ufop.br ${ }^{4}$
}

Artigo submetido em novembro/2013 e aceito em julho/2014

DOI: http://dx.doi.org/10.15628/holos.2014.1870

\section{RESUMO}

Nesse trabalho estão apresentados os resultados dos ensaios de caracterização de talco purificado de resíduos de pedra sabão, oriundos de Santa Rita de Ouro Preto, após lixiviação de concentrados de flotação com

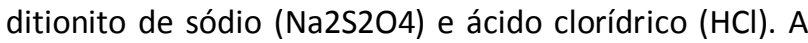
caracterização tecnológica desses materiais constou de análise granulométrica, efetuada por espalhamento de raios laser (Cilas 1064), difração de raios $X$ (método do pó total), análises químicas, efetuadas por espectroscopia ótica de emissão-plasma de acoplamento indutivo além da determinação de alvura
ISO. Para os concentrados de flotação lixiviados com ditionito de sódio e ácido clorídrico foram obtidas alvuras de 73,7 e $79,6 \%$, respectivamente, representado aumento de 3 e $9 \%$ em relação a alvura do concentrado da flotação. Verificou-se que cerca de $80 \%$ das partículas das amostras de talco lixiviadas encontravam-se abaixo de $10 \mu \mathrm{m}$. Os minerais identificados nos difratogramas de raios $\mathrm{X}$ foram talco e clinocloro. $O$ maior aumento da alvura do produto lixiviado $\mathrm{com} \mathrm{HCl}$, está relacionado com a diminuição dos teores de $\mathrm{Fe} 2 \mathrm{O} 3, \mathrm{Ni}, \mathrm{Zn}$ em relação à alimentação (concentrado da flotação).

PALAVRAS-CHAVE: Talco; pedra sabão, alvura, flotação, lixiviação

\section{CHARACTERIZATION OF PURIFIED TALC FROM WASTE OF SOAPSTONE}

\section{ABSTRACT}

This work present the results of technological characterization performed with purified talc from soapstone residues from Santa Rita de Ouro Preto, after leaching flotation concentrates with sodium dithionite and hydrochloric acid. The technological characterization consisted of size distribution performed by laser diffraction (Cilas 1064), $\mathrm{X}$ ray diffraction (total powder method), chemical analysis, performed by optical emission spectroscopy - inductively coupled plasma, density and whiteness ISO determinations. The whiteness of purified talc with sodium dithionite and hydrochloric acid were of 70.3 and $79.6 \%$, respectively. This means an increase of 3 and $9 \%$ compared with flotation concentrate whiteness. About $80 \%$ of talc particles in leached samples were smaller than $10 \mu \mathrm{m}$. The minerals talc and chlinoclore were identified in $\mathrm{X}$ ray patterns. The higher increase in whiteness with hydrochloric acid is related with the decrease of Fe2O3, $\mathrm{Ni}, \mathrm{Zn}$ compared with feed (flotation concentrate) 


\section{INTRODUÇÃO}

As rochas talcosas são provenientes de calcários e de rochas ígneas ultrabásicas com teores de $\mathrm{MgO}$ e $\mathrm{SiO}_{2}$ acima de 20 e 50\%, respectivamente. Dependendo da região podem receber denominações diferentes, esteatito, pedra sabão e saponito, que é uma rocha constituída por $35 \%$ de talco e acima de $25 \%$ dos minerais clorita, quartzo, calcita, dolomita, magnesita, tremolita, anfibólio, piroxênio e hematita (Dana, 1984, Pinheiro, 1974, Kusvart, 1984).

Na região de Ouro Preto existem diversas pedreiras de esteatito ou pedra sabão, que é extraída e exportada em forma de blocos para a Europa onde, após o desdobramento dos blocos em placas, é utilizada na construção de lareiras. Outra aplicação dessa rocha é na fabricação de ornatos em cantaria, como balaústres e colunas. É o produto mais valorizado. Além da rocha "sã" de boa qualidade são produzidas e vendidas para o artesanato (fabricação de panelas, objetos de adorno, etc.) rochas de qualidade inferior ("mais mole") e o talco, que ocorre em bolsões, que também é vendido para empresas de cerâmica.

Na tabela 1 estão apresentadas as composições químicas de oito amostras de quatro pedreiras da região de Bandeiras de Santa Rita de Ouro Preto, utilizadas em oficinas de artesanato daquela localidade, as quais produzem grande quantidade de partículas de diversos tamanhos, desde o corte das peças e durante o polimento dos objetos, que são descartados como entulho próximo aos córregos, o que posteriormente pode provocar o assoreamento dos mesmos.

Tabela 1 - Composição química de amostras de pedra sabão da região de Bandeiras - Santa Rita de Ouro Preto MG.

\begin{tabular}{|l|l|l|l|l|l|l|l|l|l|l|}
\hline Amostra & \multicolumn{9}{|l}{ Elementos (ppm) } & \multicolumn{1}{l|}{ Oxidos (\%) } \\
\hline & $\mathrm{Pb}$ & $\mathrm{As}$ & $\mathrm{Mn}$ & $\mathrm{Cu}$ & $\mathrm{SiO}_{2}$ & $\mathrm{Al}_{2} \mathrm{O}_{3}$ & $\mathrm{MgO}$ & $\mathrm{CaO}$ & $\mathrm{Fe}_{2} \mathrm{O} 3$ & $\mathrm{PPC}$ \\
\hline I & $<0,25$ & 28,83 & 881 & 53,3 & 37,12 & 3,53 & 35,23 & 1,73 & 6,71 & 15,68 \\
\hline II & $<0,25$ & 2,07 & 740 & 26,14 & 33,95 & 1,59 & 37,20 & 0,25 & 5,05 & 21,96 \\
\hline III & $<0,25$ & 1,021 & 1133 & 49,37 & 34,44 & 1,51 & 35,51 & 1,28 & 7,79 & 19,36 \\
\hline IV & $<0,25$ & $<0,05$ & 216,9 & $<0,01$ & 57,22 & 1,00 & 33,15 & 0,12 & 3,43 & 5,08 \\
\hline V & $<0,25$ & 1,638 & 742 & 40,56 & 30,99 & 0,60 & 37,24 & 0,12 & 7,54 & 23,51 \\
\hline VI & $<0,21$ & 4,349 & 1576 & 14,72 & 25,57 & 0,69 & 40,40 & 0,61 & 3,50 & 29,23 \\
\hline VII & $<0,25$ & 3,657 & 871 & 4,024 & 34,48 & 0,80 & 38,33 & 0,30 & 4,07 & 22,02 \\
\hline VIII & $<0,25$ & 12,55 & 2523 & 16,6 & 7,52 & 0,14 & 41,48 & 4,41 & 2,54 & 43,91 \\
\hline
\end{tabular}

Fonte: Lima et al. (2009).

Devido a baixa dureza e maciez do talco (1 na escala de Mohs), alta habilidade de cobertura, alto ponto de fusão, inércia química, baixa condutividade elétrica, alta capacidade de absorção de gordura, cor, óleos, resinas, baixa higroscopia e cor branca, quando puro, o mesmo é utilizado na indústria têxtil para impregnação de tecidos, indústria de sabonetes, pasta de dentes, indústria de cosméticos de modo geral, indústria de borracha (produção de tubos isolantes de fiações elétricas e na vulcanização), na indústria química como catalisador devido a grande área superficial. Quando puro é utilizado na indústria farmacêutica. No caso de pureza 
variável é uma importante matéria prima para cerâmica elétrica (Kusvard, 1984; Pontes e Almeida, 2005).

Nos ensaios de purificação de resíduos de uma oficina de artesanato, que trabalhava exclusivamente com rocha da região de Bandeiras, usando deslamagem e separação magnética, foram obtidas especificações para inseticidas e indústria têxtil, empregando flotação foram alcançadas especificações para aplicações em tintas (espalhador) e papel (carga). Após lixiviação com ácido clorídrico foram alcançadas especificações para indústria de plástico além das aplicações citadas anteriormente (Rodrigues, 2010; Rodrigues e Lima, 2012).

Nesse trabalho são apresentados os resultados de caracterização tecnológica dos concentrados de flotação após a lixiviação com ditionito de sódio e ácido clorídrico.

\section{MATERIAIS E MÉTODOS}

Neste trabalho, foi utilizada uma amostra de resíduo de pedra sabão abaixo de $74 \mu \mathrm{m}$ (200\#), proveniente de Santa Rita de Ouro Preto. A densidade da mesma era $2,9818 \mathrm{~g} / \mathrm{cm}^{3}$, área superficial igual a $2,7861 \mathrm{~m}^{2} / \mathrm{g}$, alvura ISO de $54 \%$ e $\mathrm{d}_{80}$ igual a aproximadamente $36 \mu \mathrm{m}$ (Rodrigues, 2010).

Na Figura 1 está apresentado o fluxograma dos ensaios de purificação e caracterização da amostra de resíduo de pedra sabão.

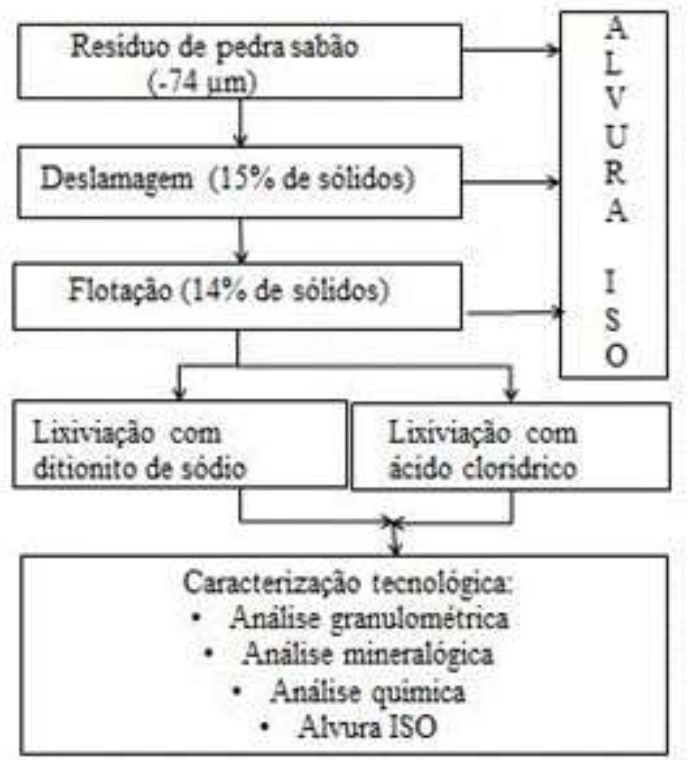

Figura 1 - Fluxograma de purificação e caracterização da amostra de resíduo de pedra sabão.

\subsection{Ensaios de Purificação}

Os ensaios de deslamagem foram efetuados da seguinte forma: transferência de $500 \mathrm{~g}$ de resíduo de pedra sabão para a cuba de $4 \mathrm{~L}$, descida do rotor da célula de flotação da marca Cimaq para a posição desejada e finalmente adicionava-se $2820 \mathrm{ml}$ de água para obtenção de uma polpa com $15 \%$ de sólidos. Posteriormente, ajustava-se a rotação da célula para $1200 \mathrm{rpm}$ e 
deixava-se a polpa sob agitação por 3 minutos. Finalmente, desligava-se a célula, levantava-se o rotor e deixava-se a polpa sedimentando por 10 minutos. Em seguida, era efetuado o sifonamento do "sobrenadante". Este procedimento era repetido por duas vezes. Finalmente, o material sifonado e afundado eram filtrados, secados a temperatura de 105 o $\mathrm{C}$ em estufa por 1 hora e meia.

Os ensaios de flotação efetuados com as amostras de resíduo previamente deslamado foram efetuadas nas condições otimizadas por Rodrigues (2010) com porcentagem de sólidos de $14 \%, 500 \mathrm{~g} / \mathrm{t}$ de metassilicato de sódio e $150 \mathrm{~g} / \mathrm{t}$ de metil isobutil carbinol (MIBC).

O preparo das soluções de reagentes (metassilicato de sódio e MIBC) utilizadas nos ensaios de flotação foi efetuado da seguinte forma:

i. para o preparo da solução de metassilicato de sódio a 0,5\% p/v, pesou-se $0,5 \mathrm{~g}$ de metassilicato que foi diluído em $100 \mathrm{ml}$ de água destilada em um balão volumétrico;

ii. para o preparo da solução de $M I B C$ a $0,25 \% \mathrm{p} / \mathrm{v}$, pesou-se $0,25 \mathrm{~g}$ de $\mathrm{MIBC}$ e diluindo-o com água destilada em balão volumétrico de $100 \mathrm{ml}$.

Os ensaios de lixiviação do produto flotado com ditionito de sódio e ácido clorídrico foram efetuados nas condições otimizadas por Rodrigues (2010) conforme descrito abaixo:

a) Ditionito de sódio: uma solução com concentração de $\mathrm{Na}_{2} \mathrm{~S}_{2} \mathrm{O}_{4}$ a $1 \%$ p/v, 20\% de sólidos e tempo de lixiviação igual a 120 minutos. O procedimento adotado foi o seguinte:

i. ipreparar a solução de ditionito de sódio a $1 \% \mathrm{p} / \mathrm{v}$, colocando $2,5 \mathrm{~g}$ de diotionito de sódio em um balão de $250 \mathrm{ml}$ completando-o com água destilada;

ii. introduzir $23 \mathrm{~g}$ de talco purificado por flotação em um béquer de $200 \mathrm{ml}$ e adicionar $87 \mathrm{ml}$ de água destilada em pH 5 (ajustado com solução de ácido sulfúrico) e $6 \mathrm{ml}$ de solução de ditionito de sódio ficando com uma suspensão com $20 \%$ de sólidos;

iii. verificar o valor do $\mathrm{pH}$ e deixar agitar em uma rotação de $600 \mathrm{rpm}$ por 120 minutos em temperatura ambiente;

iv. efetuar a filtragem e secar o produto a uma temperatura aproximadamente de $105^{\circ} \mathrm{C}$ por 1 hora e meia.

b) Ácido clorídrico

Uma solução de $\mathrm{HCl}$ a $4 \mathrm{M}$, temperatura de $80^{\circ} \mathrm{C}$, porcentagem de sólidos de $15 \%$ e tempo de lixiviação igual a 60 minutos. $O$ procedimento adotado foi o seguinte:

i. preparar uma solução de $\mathrm{HCl}$ a $4 \mathrm{M}$ colocando $168 \mathrm{ml}$ de $\mathrm{HCl}$ em um balão volumétrico de $500 \mathrm{ml}$ completando-o com água destilada;

ii. transferir $95 \mathrm{ml}$ da solução de $\mathrm{HCl}$ a $4 \mathrm{M}$ para um béquer contendo $17 \mathrm{~g}$ de talco purificado por flotação, formando uma suspensão com $15 \%$ de sólidos; 
iii. aquecer essa suspensão até $80^{\circ} \mathrm{C}$. Logo em seguida agitar em uma rotação de aproximadamente $700 \mathrm{rpm}$ por 60 minutos. Sendo a temperatura verificada por um termômetro nesse período, variando-a em uma faixa entre 78 e $82{ }^{\circ} \mathrm{C}$;

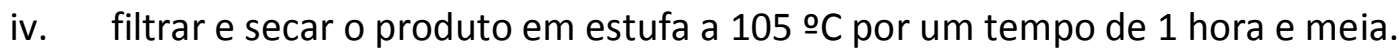

\subsection{Caracterização Tecnológica}

Os ensaios de caracterização física das amostras lixiviadas constaram de análise granulométrica utilizando granulômetro a laser CILAS 1064, determinação de densidade usando um picnômetro a gás Ultrapcnometer 1000 e determinação da área superficial das partículas usando o equipamento BET Nova 1200 do Laboratório de Propriedades Interfaciais do Departamento de Engenharia de Minas da UFOP.

A caracterização mineralógica foi feita através da difração de raios $X$ (método do pó total). Para a obtenção dos difratogramas de raios $X\left(2^{\circ}\right.$ a $\left.70^{\circ}\right)$, foi utilizado o difratômetro de raios $X$ tipo 11076186 da PanAnalytical do Laboratório de Difração de Raios X do DEGEO/UFOP. A radiação utilizada foi $\mathrm{K}_{\alpha \mathrm{Cu}}=1,54 \AA$.

As análises químicas das amostras foram efetuadas por espectrofotometria de emissão atômica com fonte de plasma (ICP-OES) modelo Ciros CCD da marca Spectro do Laboratório de Geoquímica do Departamento de Geologia/UFOP. Os elementos químicos analisados foram Al, As, $\mathrm{Ca}, \mathrm{Cu}, \mathrm{Fe}, \mathrm{Mg}$, Ni, Pb, Ti e $\mathrm{Zn}$. O teor de $\mathrm{SiO}_{2}$ foi obtido por diferença.

Para determinação de alvura ISO dos produtos da deslamagem, flotação e das amostras lixiviadas foi utilizado o espectrofotômetro Color Touch model do Laboratório de Propriedades Interfaciais do DEMIN/UFOP. O procedimento para a confecção das pastilhas para medida de alvura das amostras constou de:

i. quartear o produto até obter uma alíquota de aproximadamente $50 \mathrm{~g}$;

ii. secar a amostra em estufa a uma temperatura aproximadamente de $105^{\circ} \mathrm{C} \mathrm{em}$ um vidro relógio;

iii. pulverizar a amostra por 1 minuto em um moinho orbital, usando panela de tungstênio;

iv. confeccionar um corpo de prova para o teste, utilizando a prensa Powder Press Apparatus.

Essa confecção foi feita em um pequeno cilindro oco, que era colocado em cima de uma superfície lisa de vidro sobre uma balança, em seguida colocou-se o material no cilindro e aplicou-se uma força de aproximadamente 56,70 kgf.

\section{RESULTADOS E DISCUSSÃO}

Observa-se pela Figura 2 que não houve diferença entre as distribuições granulométricas das amostras de talco lixiviado com ditionito de sódio e com ácido clorídrico. Ou seja, o $d_{80}$ das mesmas foi de aproximadamente $75 \mu \mathrm{m}$ e que cerca de $10 \%$ das partículas encontravam-se abaixo de $10 \mu \mathrm{m}$. 
Na Tabela 2 estão apresentadas as composições químicas e a alvura do produto afundado, das amostras lixiviadas com ditionito de sódio e com ácido clorídrico.

As densidades das amostras de talco lixiviadas com Na${ }_{2} \mathrm{~S}_{2} \mathrm{O}_{4}$ e com $\mathrm{HCl}$ foram de 2,8929 $\mathrm{g} / \mathrm{cm}^{3}$ e $2,9471 \mathrm{~g} / \mathrm{cm}^{3}$, respectivamente. Estes valores estão dentro das especificações para a aplicação em tintas $\left(2,8\right.$ a $\left.2,9 \mathrm{~g} / \mathrm{cm}^{3}\right)$ e são ligeiramente menores do que o valor de densidade da amostra de resíduo de pedra sabão $\left(2,9818 \mathrm{~g} / \mathrm{cm}^{3}\right)$, provavelmente, devido à remoção parcial de sulfetos e magnetita, que são minerais de densidades mais altas. As áreas superficiais foram de $1,8259 \mathrm{~m}^{2} / \mathrm{g}$ e $2.6639 \mathrm{~m}^{2} / \mathrm{g}$, respectivamente para as amostras de talco, previamente purificadas por flotação, lixiviadas com $\mathrm{Na}_{2} \mathrm{~S}_{2} \mathrm{O}_{4}$ e $\mathrm{HCl}$, respectivamente. Esta diferença entre os valores medidos não está coerente com as distribuições granulométricas, que foram praticamente iguais para as duas amostras (Figura 2). No entanto, pode-se afirmar que os menores valores das áreas superficiais das amostras lixiviadas em relação à alimentação $\left(2,7861 \mathrm{~m}^{2} / \mathrm{g}\right)$ estão relacionados com a distribuição granulométrica $\left(\mathrm{d}_{80}=74 \mu \mathrm{m}\right)$ das mesmas serem mais grosseiras do que da alimentação $\left(\mathrm{d}_{80}=36 \mu \mathrm{m}\right)$, devido à remoção das partículas finas com a operação de deslamagem antes da flotação. No entanto, há de salientar que a análise granulométrica da alimentação foi efetuada por peneiramento e dos produtos lixiviados por granulômetro a laser, cujos valores de tamanho de partículas, normalmente são maiores do que os tamanhos medidos por peneiramento.

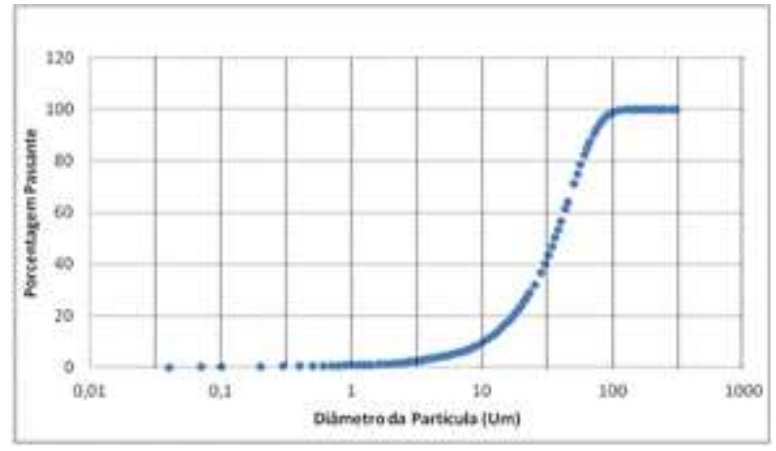

(a)

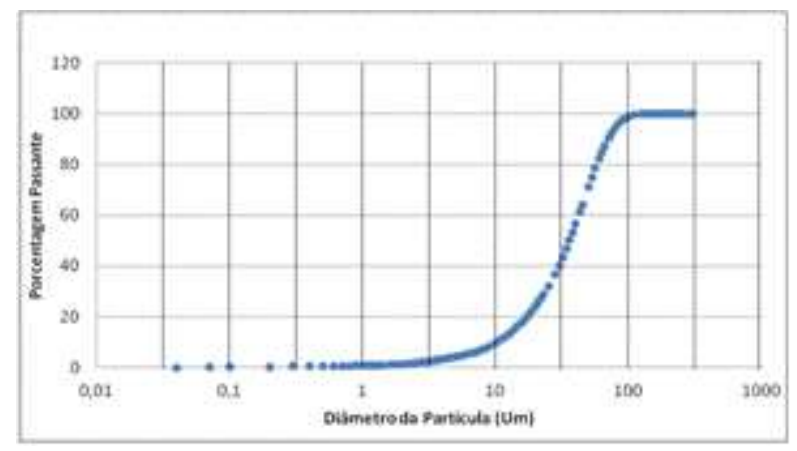

(b)

Figura 2 - Distribuição granulométrica da amostra de talco lixiviada com ditionito de sódio (a) e ácido clorídrico (b)

Tabela 2 - Composição química das amostras de talco lixiviadas com ditionito de sódio e com ácido clorídrico.

\begin{tabular}{|c|c|c|c|c|}
\hline \multicolumn{2}{|c|}{ Teor } & \multicolumn{3}{c|}{ Amostra de talco } \\
\cline { 3 - 5 } \multicolumn{6}{c|}{} & Alimentação & Lixiviado com $\mathrm{Na}_{2} \mathrm{~S}_{2} \mathrm{O}_{4}$ & Lixiviado com $\mathrm{HCl}$ \\
\hline \multirow{4}{*}{$(\%)$} & $\mathrm{Al}_{2} \mathrm{O}_{3}$ & 2,27 & 1,355 & 0,013 \\
& $\mathrm{MgO}$ & 27,7 & 31,41 & 30,11 \\
& $\mathrm{CaO}$ & 0,580 & 0,028 & 0,036 \\
& $\mathrm{TiO}_{2}$ & 0,051 & 0,054 & 0,050 \\
& $\mathrm{Fe}_{2} \mathrm{O}_{3}$ & 4,7 & 3,43 & 3,55 \\
& $\mathrm{SiO}_{2}$ & 58,55 & 57,42 & 59,78 \\
& $\mathrm{PPC}$ & 5,98 & 6,12 & 6,28 \\
\hline \multirow{4}{*}{$(p p m)$} & $\mathrm{Ni}$ & 1956 & 1727 & 1715 \\
& $\mathrm{Cu}$ & 13,4 & - & - \\
& $\mathrm{Zn}$ & 85,8 & 65,8 & 59,3 \\
& $\mathrm{As}$ & 11,1 & - & - \\
\hline \multicolumn{2}{|l}{ Densidade $\left(\mathrm{g} / \mathrm{cm}^{3}\right)$} & 2,982 & 2,893 & 2,947 \\
\hline
\end{tabular}




\begin{tabular}{|l|c|c|c|}
\hline Alvura \% (ISO) & 54,00 & 73,7 & 79,6 \\
\hline
\end{tabular}

*Alvura ISO do produto flotado - 70,9\%

**Alvura ISO do produto afundado $-59,5$

Lima et al. (2012) através de difratometria de raios $X$ dos resíduos de pedra sabão da região de Bandeiras identificaram os seguintes minerais: talco $\left(\mathrm{Mg}_{3} \mathrm{SiO}_{4} \mathrm{O}_{10}(\mathrm{OH})_{2}\right.$, caolinita $\left(\mathrm{SiO}_{2} \mathrm{Al}_{2} \mathrm{O}_{5}(\mathrm{OH})_{4}\right.$, clorita $\left((\mathrm{Mg}, \mathrm{Al})_{6}(\mathrm{Si}, \mathrm{Al})_{4} \mathrm{O}_{10}(\mathrm{OH})_{8}\right)$ e nimita $((\mathrm{Ni}, \mathrm{Mg}, \mathrm{Al})(\mathrm{Si}, \mathrm{Al}) \mathrm{O}(\mathrm{OH})$ em todas as faixas granulométricas analisadas. Através de microspia ótica e microscopia eletrônica de varredura de amostras de pedra sabão de Bandeiras, Lima et al. (2009) identificaram sulfetos: pirita $\left(\mathrm{FeS}_{2}\right)$, arsenopirita ( $\left.\mathrm{FeAsS}\right)$, pentlandita $\left((\mathrm{Fe}, \mathrm{Ni})_{9} \mathrm{~S}_{8}\right)$; carbonatos: magnesite $\left(\mathrm{MgCO}_{3}\right)$, dolomite $\left(\mathrm{CaMg}\left(\mathrm{CO}_{3}\right)_{2}\right)$ e óxidos: magnetita/hematita $\left(\mathrm{Fe}_{3} \mathrm{O}_{4} / \mathrm{F}_{2} \mathrm{O}_{3}\right)$, ilmenita $\left(\mathrm{TiO}_{2}\right)$. Logo, os teores dos compostos e elementos químicos presentes na alimentação e nos produtos lixiviados estão coerentes com a mineralogia de amostras de pedra sabão da região de Bandeiras.

Nos difratogramas de raios $\mathrm{X}$ das amostras lixiviadas tanto com ditionito de sódio e como com ácido clorídrico foram identificados os minerais talco e clinocloro. Na Figura 3 está apresentado o difragrama típico das amostras lixiviadas, com os picos principais dos minerais identificados. Os demais picos são atribuídos à superposição de picos dos dois minerais identificados. A não identificação dos demais minerais identificados em trabalhos prévios (Lima et al., 2012) pode estar relacionada com o baixíssimo teor dos minerais nas amostras analisadas.

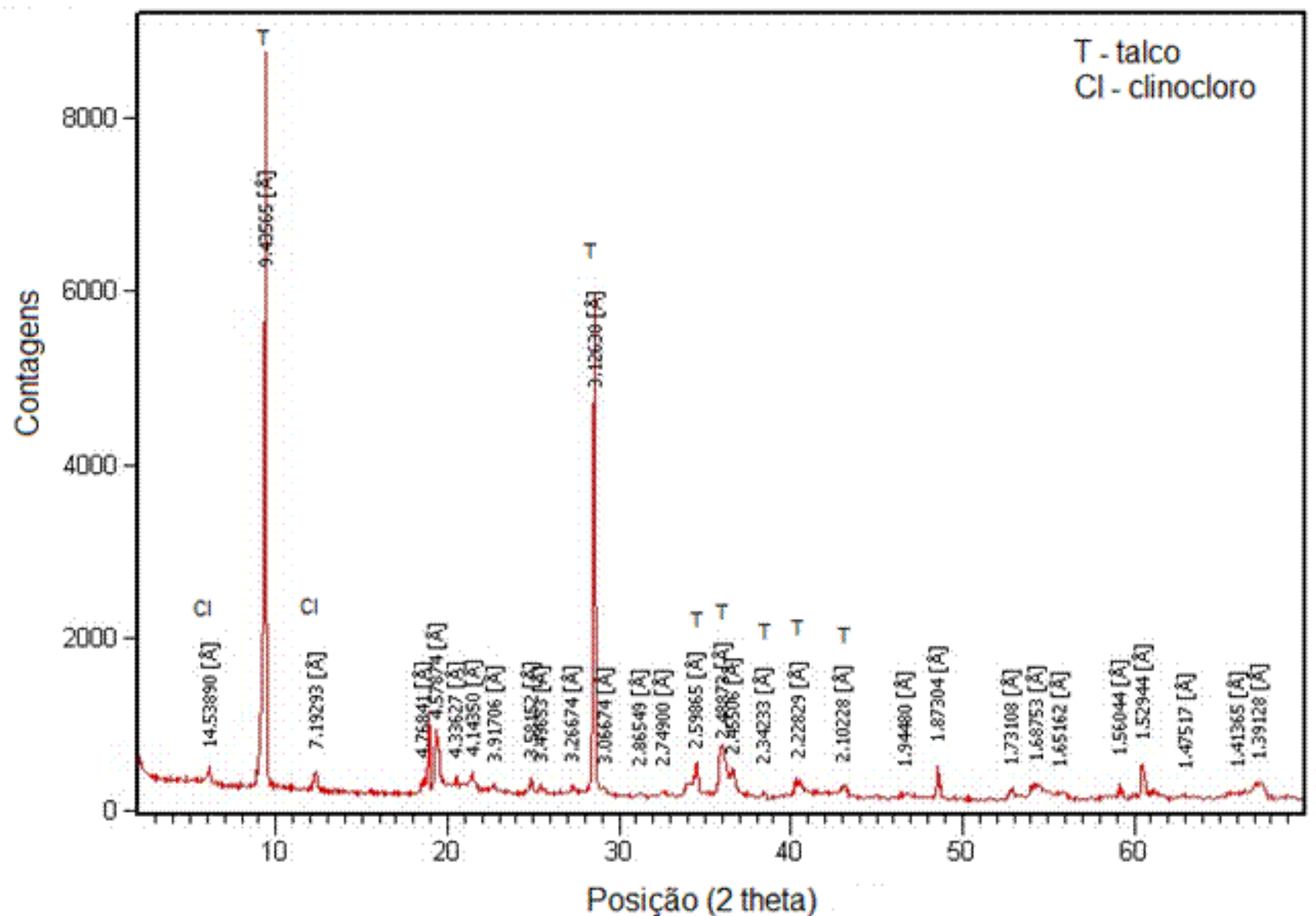

Figura 3 - Difratograma de raios X típico das amostras lixiviadas. 
Observa-se pela Tabela 2 diminuição acentuada do teor de $\mathrm{Al}_{2} \mathrm{O}_{3}$ em relação à alimentação, que provavelmente está relacionado com a remoção de caolinita na deslamagem do material e remoção de nimita pela lixiviação com $\mathrm{HCl}$, uma vez que não foi identificado este mineral na difração de raios $X$ dos produtos lixiviados. Observa-se também que houve pequena remoção de pentlandita da amostra, uma vez que a redução do teor de $\mathrm{Zn}$ foi pequena em relação a teor de Zn na alimentação.

A diminuição do teor de $\mathrm{Fe}_{2} \mathrm{O}_{3}$ no produto lixiviado com ditionito de sódio e consequente aumento da alvura em relação à alimentação (produto flotado), está relacionado com a redução do $\mathrm{Fe}^{3+}$ da magnetita para $\mathrm{Fe}^{2+}$ (Luz, et al., 1990). Pela redução dos teores de $\mathrm{CaO}$ e $\mathrm{MgO}$ e aumento considerável da alvura com lixiviação da amostra com $\mathrm{HCl}$, pode-se inferir que houve remoção de carbonatos e sulfetos da amostra lixiviada (Ahmed et al., 2007).

Pelos resultados de teores e alvura dos produtos lixiviados foram alcançadas especificações para as seguintes aplicações: inseticida (granulometria abaixo de $43 \mu \mathrm{m}$ ), tintas pigmentos (alvura 77 a 87\%, MgO: 24 a $32 \%, \mathrm{SiO}_{2}: 50$ a $65 \%, \mathrm{CaO} \leq 9 \%, \mathrm{MgO}+\mathrm{SiO}_{2} \geq 88 \%$, $\mathrm{CO}_{2} \leq 1 \% \mathrm{Al}_{2} \mathrm{O}_{3}+\mathrm{Fe}_{2} \mathrm{O}_{3} \leq 6 \%, \mathrm{H}_{2} \mathrm{O}+\mathrm{MV} \leq 1 \% \mathrm{PPC} \leq 7 \%$, peso específico entre 2,8 a $2,9 \mathrm{~g} / \mathrm{cm}^{3}$ ) e espalhador (granulometria 100\% abaixo de $74 \mu \mathrm{m}$ e $96,5 \%$ abaixo de $43 \mu \mathrm{m}$, alvura -65 a $87 \%$, $\mathrm{SiO}_{2}+\mathrm{MgO} \geq 75 \% \mathrm{Al}_{2} \mathrm{O}_{3} \leq 2 \% \mathrm{H}_{2} \mathrm{O}+\mathrm{M}$.Vol. $<1 \%$, PPC- $\leq 7 \%$, peso específico entre 2,8 e $2,9 \mathrm{~g} / \mathrm{cm}^{3}$ e partículas lamelares (Rodrigues e Lima, 2012 e 2011)), carga para papel (95\% menor que $37 \mu \mathrm{m}$, alvura 54 e $75 \%$ ) e plástico (100\% menor que $74 \mu \mathrm{m}$ e alvura superior a $74 \mu \mathrm{m}$ ) (Pontes e Almeida, 2005).

\section{CONCLUSÕES}

A distribuição granulométrica dos produtos lixiviados foi maior e consequentemente, as áreas superficiais dos mesmos foram maiores do que da alimentação, provavelmente devido à remoção das partículas finas pela deslamagem, especialmente a caolinita. A menor densidade dos produtos lixiviados em relação à alimentação está relacionada com a remoção de sulfetos. Os minerais identificados nos difratogramas das amostras lixiviadas foram talco e clinocloro. Levando em consideração os teores obtidos após lixiviação do produto flotado com ditionito de sódio verificou-se aumento da alvura do produto lixiviado de cerca de $3 \%$ em relação ao produto flotado (70,9\%), que está relacionado à remoção da magnetita, que ocorre pela redução de $\mathrm{Fe}^{3+}$ para $\mathrm{Fe}^{2+}$. Na lixiviação do produto flotado com ácido clorídrico foi alcançada alvura de $79,6 \%$, que está relacionada com a solubilização e remoção de sulfetos e carbonatos, presentes no material. Com esses valores de alvura o talco pode ser aplicado para fabricação de papel (carga), tintas (pigmento) e plástico.

\section{AGRADECIMENTOS}

Os autores agradecem à Fapemig pelo apóio financeiro, à CAPES pela bolsa de mestrado, ao CNPq pela bolsa de produtividade em pesquisa e à UFOP pela bolsa de iniciação científica.

\section{REFERÊNCIAS BIBLIOGRÁFICAS}

1. AHMED, M. M., IBRAHIM, G. A., HASSAN, M. M.A. Improvement of Egyptian talc quality for 
industrial uses by flotation process and leaching. International Journal of Mineral Processing. 83, 132-145. 2007.

2. DANA, J. D., HURLBULT, C. S. In: Manual de Mineralogia, Livros Técnicos e Científicos Editora S.A., v. II, Rio de Janeiro - RJ, p.642, 1984.

3. KUZVART, M. Industrial Minerals and Rocks. Elsevier. Amsterdam-Oxford-New York-Tokyo. 1984. 454p.

4. LIMA, R. M. F., BRAGA, D. M., SILVA, F. B. R. Technological Characterization of Soap Stone from Santa Rita de Ouro Preto/Brazil. Proceedings of the $V$ International Materials Symposium, Lisboa, Portugal, p. 1-6, 2009.

5. PINHEIRO, J.C.F. Perfil Analítico do Talco, Departamento Nacional de Produção Mineral, Boletim 22 - Ministério das Minas e Energia. Rio de Janeiro, BRASIL, 1974, p.41.

6. PONTES, I. F., ALMEIDA, S. L. M. Talco. In: Rochas e minerais industriais usos e especificações. LUZ, A. B., LINS, F. F. (Ed.). Rio de Janeiro: CETEM/MCT. 2005. 623p.

7. RODRIGUES, M. L. M. Caracterização Tecnológica de Resíduos de Pedra Sabão de Oficinas de Artesanato em Pedra Sabão da Região de Ouro Preto. Ouro Preto: Programa de PósGraduação em Engenharia Mineral, Universidade Federal de Ouro Preto, 2010. 137p. (Dissertação de Mestrado).

8. RODRIGUES, M. L. M., LIMA, R. M. F., Cleaner production of soapstone in the Ouro Preto region of Brazil: a case study. Journal of Cleaner Production. 32, 2012, p. 149-156. 